\title{
Risiken und Nebenwirkungen der Informatisierung des Alltags
}

\author{
Hilty, Lorenz
}

\begin{abstract}
Die "Informatisierung des Alltags" ist eine Vision mit einem hohen gesellschaftlichen Veränderungspotenzial. Die Abschätzung der Folgen einer sol-chen Entwicklung ist notwendig, will man unerwartete negative Auswirkungen minimieren. In der kurzen Geschichte des breiten Einsatzes digitaler Informations- und Kommunikationstechnologien hat es sich bereits gezeigt, dass sich der technische Fortschritt nicht automatisch in die erhofften Vorteile für Individuum, Orga-nisation und Gesellschaft übersetzt. Beispielsweise muss der Gebrauch schnellerer Hardware nicht zu einer höheren persönlichen Arbeitseffizienz führen und der Einsatz von IKT in Organisationen nicht zu geringeren Informationskosten. Dieses "IT productivity paradox" ist teilweise durch Rebound-Effekte zu erklären, die aus einer technikzentrierten Perspektive meist übersehen werden. Psychologische, gesundheitliche, soziale und ökonomische Effekte einer höheren technischen Effizienz müssen daher in einer Abschätzung der Technologiefolgen berücksichtigt werden. Eine Prospektivstudie fü;r Pervasive Computing hat gezeigt, dass jenseits von Produktivitätsaspekten auch die Problemfelder Stress (u.a. durch das Gefühl des Überwachtwerdens), Freiwilligkeit (Autonomie von Konsumenten oder Patienten), unbeherrschbare Komplexität (emergente Eigenschaften der entstehenden Infrastruktur) und Fragen der ökologischen Nachhaltigkeit zu beachten sind.
\end{abstract}

Posted at the Zurich Open Repository and Archive, University of Zurich

ZORA URL: https://doi.org/10.5167/uzh-58873

Book Section

Published Version

Originally published at:

Hilty, Lorenz (2007). Risiken und Nebenwirkungen der Informatisierung des Alltags. In: Mattern, Friedemann. Der Computer im 21. Jahrhundert. Die Informatisierung des Alltags. Perspektiven, Technologien, Auswirkungen. Berlin: Springer, 187-205. 


\section{REPRINT}

\section{Risiken und Nebenwirkungen der}

\section{Informatisierung des Alltags}

Lorenz M. Hilty

Dieser Beitrag ist erschienen in:

Mattern, F. (Hrsg.): Der Computer im 21. Jahrhundert. Die Informatisierung des Alltags.

Perspektiven, Technologien, Auswirkungen. Springer-Verlag, Berlin 2007, S. 187-205

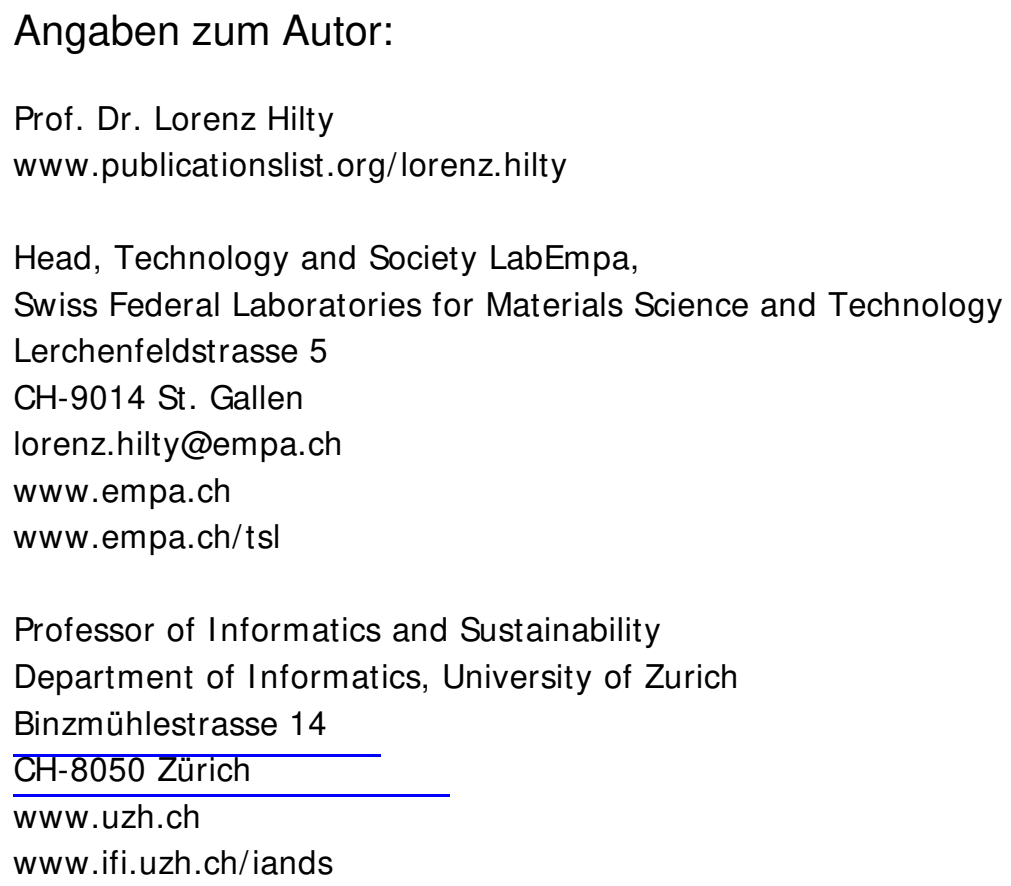




\title{
Risiken und Nebenwirkungen der Informatisierung des Alltags
}

\author{
Lorenz M. Hilty \\ Abteilung Technologie und Gesellschaft, Empa, St.Gallen, Switzerland
}

Kurzfassung. Die „Informatisierung des Alltags“ ist eine Vision mit einem hohen gesellschaftlichen Veränderungspotenzial. Die Abschätzung der Folgen einer solchen Entwicklung ist notwendig, will man unerwartete negative Auswirkungen minimieren. In der kurzen Geschichte des breiten Einsatzes digitaler Informationsund Kommunikationstechnologien hat es sich bereits gezeigt, dass sich der technische Fortschritt nicht automatisch in die erhofften Vorteile für Individuum, Organisation und Gesellschaft übersetzt. Beispielsweise muss der Gebrauch schnellerer Hardware nicht zu einer höheren persönlichen Arbeitseffizienz führen und der Einsatz von IKT in Organisationen nicht zu geringeren Informationskosten. Dieses „IT productivity paradox“ ist teilweise durch Rebound-Effekte zu erklären, die aus einer technikzentrierten Perspektive meist übersehen werden. Psychologische, gesundheitliche, soziale und ökonomische Effekte einer höheren technischen Effizienz müssen daher in einer Abschätzung der Technologiefolgen berücksichtigt werden. Eine Prospektivstudie für Pervasive Computing hat gezeigt, dass jenseits von Produktivitätsaspekten auch die Problemfelder Stress (u.a. durch das Gefühl des Überwachtwerdens), Freiwilligkeit (Autonomie von Konsumenten oder Patienten), unbeherrschbare Komplexität (emergente Eigenschaften der entstehenden Infrastruktur) und Fragen der ökologischen Nachhaltigkeit zu beachten sind.

\section{Einleitung}

Die politische Vision einer Informationsgesellschaft, deren Wertschöpfungsprozesse sich als Folge des allgemeinen Zugangs zu Informations- und Kommunikationstechnologien (IKT) grundlegend von denen der Industriegesellschaft unterscheiden, verbindet sich heute mit der technologischen Vision einer Informatisierung des Alltags. Gegenstände des täglichen Gebrauchs sollen zu „smart objects“ werden und selbsttätig Daten austauschen können. Nach der,,letzten Meile“ zum Hausanschluss sollen nun die letzten Meter und Zentimeter zu den Alltagsgegenständen im Wohnzimmer, am Arbeitsplatz oder am Handgelenk digital überbrückt werden.

Diese Visionen besitzen gesellschaftliches Veränderungspotenzial. Neue Geschäftsmodelle, aber auch neue Formen von Konflikten werden voraussichtlich als emergente Phänomene aus der entstehenden Infrastruktur hervorgehen. Die Bedeutung des physikalisch-geographischen Raumes als Ordnungsprinzip für Ursache und Wirkung wird schrittweise abnehmen. Andere Ordnungsprinzipien wie die 
Topologie von Netzwerken, Zugriffsrechte auf Datenbestände und geistige Eigentumsrechte werden langfristig bedeutsamer werden als die Kontrolle über ein physisches Territorium. Angesichts dieses Veränderungspotenzials erscheint es notwendig, auch über mögliche Nebenwirkungen und Risiken einer „Informatisierung des Alltags" nachzudenken.

In der Medizin bezeichnet man als Nebenwirkung einer Behandlungsmethode einen Effekt, der zwar unerwünscht ist, aber zugunsten des beabsichtigten Haupteffekts häufig in Kauf genommen wird. Der Patient muss also zwischen Hauptund Nebenwirkung abwägen.

Ein Risiko ist ein unerwünschter Effekt, der nicht mit Sicherheit eintritt. Der mögliche Schaden kann hier wesentlich höher sein als der erwartete Nutzen der Behandlung, jedoch tritt er nur mit einer geringen Wahrscheinlichkeit ein. Hier ist also der erwartete Nutzen gegen das quantifizierte Risiko (Schadenshöhe multipliziert mit Eintrittswahrscheinlichkeit) abzuwägen. Häufig ist das Risiko allerdings nicht quantifizierbar, weil die Wahrscheinlichkeit nicht bekannt ist oder weil der Schaden aus prinzipiellen Gründen nicht in Zahlen auszudrücken ist - die Abwägung ist dann notwendigerweise subjektiv.

Entlang dieser medizinischen Analogie will ich in diesem Beitrag einige kritische Fragen an die Vision bzw. die Visionäre der „Informatisierung des Alltags“ richten. Dabei bin ich mir bewusst, dass die Analogie ihre Grenzen hat: Während das Ziel einer medizinischen Behandlung klar definiert ist - eine bestimmte Erkrankung zu vermeiden, zu heilen oder zu lindern -, so ist das gesellschaftliche Ziel eines ubiquitären Einsatzes von IKT nicht vorgegeben. Es fehlt also ein verbindlicher Konsens über den Nutzen, gegen den die Nebenwirkungen und Risiken abzuwägen wären.

Wie der UN-Weltgipfel zur Informationsgesellschaft in Tunis 2005 erneut gezeigt hat, ist ein gemeinsamer Nenner über den nutzbringenden Einsatz von IKT meist nur in relativ abstrakten Bekenntnissen wie „Armutsbekämpfung“ oder „Zugang für alle" zu finden. Solche Ziele klingen positiv, sind aber aufgrund ihres hohen Abstraktionsniveaus nahezu inhaltsleer. Der politische Konsens wird schnell brüchig, wenn es darum geht, die Ziele zu konkretisieren.

Im anschließenden, zweiten Abschnitt dieses Beitrages werde ich deshalb einige der bisher mit dem Einsatz von IKT verfolgten Ziele (,Effizienz“ und „Produktivität"“) als gegeben betrachten und zunächst untersuchen, wie weit diese in der Vergangenheit realisiert werden konnten. Meine Hauptthese ist hier, dass es uns in den vergangenen Jahrzehnten relativ schlecht gelungen ist, Fortschritte im Bereich der IKT in Fortschritte für Menschen und Organisationen zu übersetzen. Aus heutiger Sicht ist das u.a. dadurch zu erklären, dass systemische Nebenwirkungen des IKTEinsatzes übersehen wurden. Wenn aber schon diese vergleichsweise bescheidenen Ziele nicht erreicht werden konnten, was ist denn von den heutigen, viel weitergehenden Zukunftsvisionen zu halten?

Der dritte Abschnitt konzentriert sich auf die Risiken einer möglichen zukünftigen „Informatisierung des Alltags“ und greift das oben erwähnte Problem wieder auf, nicht quantifizierbare Risiken in Entscheidungen einbeziehen zu müssen. Vor dem Hintergrund des Vorsorgeprinzips werden hier qualitative Kriterien zur Risikocharakterisierung herangezogen. Der Abschnitt fasst die Ergebnisse einer Studie 
zusammen, die die Empa ${ }^{1}$ gemeinsam mit dem Institut für Zukunftsstudien und Technologiebewertung (IZT), Berlin, für das Schweizerische Zentrum für Technologiefolgen-Abschätzung erstellt hat [HBB03]. Diese Studie hat unter anderem gezeigt, dass neben unbestrittenen Chancen einer totalen Vernetzung des Alltags für Gesellschaft und Umwelt auch Risiken zu beachten sind, die mit technikbedingtem Stress, mit Fragen der Autonomie und Freiwilligkeit und mit unbeherrschbarer Komplexität in Zusammenhang stehen. Nicht zuletzt wird die zukünftige Anwendung von IKT auch die Möglichkeit einer nachhaltigen Entwicklung maßgeblich beeinflussen.

\section{Lehren aus der Vergangenheit}

Unerwartete, scheinbar paradoxe Phänomene sind die besten Ausgangspunkte für ein vertieftes Verständnis komplexer Systeme. In diesem Abschnitt sind unter diesem Gesichtspunkt einige Ergebnisse zu den Wechselwirkungen zwischen IKT und ihren Benutzer/innen im Kontext von Organisationen zusammengestellt. Der gemeinsame Nenner der zitierten Ergebnisse ist der Rebound-Effekt, eine ökonomische Erklärung der häufig kontra-intuitiven Auswirkungen technischer Effizienz. Für eine zukünftige „Informatisierung des Alltags“ wird es entscheidend sein, diese (und ähnliche) Effekte realistisch einzuschätzen, um Wege zu ihrer Vermeidung zu finden.

\subsection{Vorbemerkungen zu Effizienz und Rebound-Effekt}

Effizienz im weitesten Sinn ist das Verhältnis von Ergebnis (Output) zu benötigten Ressourcen (Input). Verdoppelt sich die Effizienz eines Vorgangs, so kann doppelt so viel Output mit gleich viel Input erzeugt werden wie zuvor, oder auch der gleiche Output mit der Hälfte des Inputs. Die Effizienz gibt lediglich das Verhältnis an. Im Gegensatz zu Effektivität ist Effizienz immer eine Verhältniszahl.

Abhängig davon, welche Ressourcen man auf der Inputseite betrachtet, kann man verschiedene Arten von Effizienz definieren. Betrachtet man beispielsweise nur die Ressource „Zeitaufwand“, so kann man von „Zeiteffizienz“ sprechen. Andere Arten von Effizienz ergeben sich, wenn man statt Zeit etwa den Input an Energie betrachtet (Energieeffizienz), oder den Materialverbrauch (Materialeffizienz), den benötigten Raum (Raumeffizienz) oder das Geld, das ausgegeben werden muss (Kosteneffizienz).

Die IKT hat fast unglaubliche Fortschritte in allen genannten Formen von Effizienz gemacht, wenn man die Hardware-Leistung als Output betrachtet. ${ }^{2}$

Eidgenössische Materialprüfungs- und Forschungsanstalt

2 Hinter dieser Entwicklung steht das so genannte Moore'sche Gesetz, dessen genaue Bedeutung und empirische Gültigkeit hier aber nicht thematisiert werden sollen (vgl. aber [Mat03]). 
Schon in den 1980er-Jahren hat die amerikanische Presse die viel zitierte Analogie zwischen IKT und Automobil populär gemacht: Wenn die Automobilindustrie die gleichen Fortschritte gemacht hätte wie die Computerindustrie, hätte ein Rolls-Royce damals 2.50 Dollar gekostet und mit einer Tankfüllung zwei Millionen Meilen zurückgelegt. Heute, im Jahr 2006, würde der Rolls-Royce nur noch wenige Cents kosten und wahrscheinlich als Werbegeschenk verteilt werden. Und das Nachfüllen des Tanks wäre nicht vorgesehen.

Aus der Ökonomie ist bekannt, dass Effizienzverbesserungen häufig einen so genannten Rebound-Effekt nach sich ziehen. Das bedeutet, dass eingesparte Ressourcen (Zeit, Geld, Energie, ...) unter bestimmten Voraussetzungen zu einer Ausweitung der Nachfrage führen, so dass absolut gesehen keine Einsparung eintritt. Wenn die Mengenausweitung die mögliche Ressourceneinsparung exakt kompensiert, spricht man von einem Rebound-Effekt von $100 \%$.

Das bekannteste Beispiel hierfür ist die historische Verkehrsentwicklung (oder auch der heutige Vergleich des Verkehrs zwischen Ländern mit unterschiedlichem Entwicklungsstand). Bedingt durch den technischen Fortschritt sind die Verkehrsträger in den letzten 200 Jahren immer schneller geworden, d.h. eine bestimmte Distanz kann in immer kürzerer Zeit zurückgelegt werden. Die Zeiteffizienz wurde somit stark erhöht. Das hat jedoch nicht zu Zeitersparnissen geführt, denn jede Effizienzerhöhung wurde sofort durch vermehrtes Reisen kompensiert. Der Rebound-Effekt beträgt hier ungefähr $100 \%$ und ist auch als „Constant Travel Time Hypothesis“ bekannt: Weltweit reisen die Menschen ungefähr 70 Minuten pro Tag (Jahresdurchschnitt pro Person) und zwar sowohl in Tansania als auch in den USA. Das Einzige, was sich mit dem technischen Fortschritt und den damit verbundenen Effizienzverbesserungen ändert, ist die pro Kopf zurückgelegte Distanz, die in den USA um ein Vielfaches höher ist als in Tansania. Dieser Rebound-Effekt führt unter anderem dazu, dass der Energieverbrauch des Verkehrs ständig zunimmt, da dank schnelleren Transportmitteln in der gleichen Zeit immer mehr bzw. immer weiter gereist werden kann.

Ein weiteres Beispiel ist der Ersatz herkömmlicher Korrespondenz durch EMail. E-Mail ist zweifellos eine wesentlich zeiteffizientere Kommunikationsmethode, weil das Ausdrucken eines Bogens, das Kopieren von Beilagen, das Hantieren mit Briefumschlägen usw. entfällt. Dennoch zeigt die Alltagserfahrung, dass wir heute mehr Zeit für E-Mails aufwenden als früher mit dem Lesen und Schreiben von Briefen, weil die Anzahl der Nachrichten pro Zeiteinheit stark angewachsen ist. Dies liegt zum einen an der der Möglichkeit, mit gleichem Zeitaufwand mehr Kontakte zu unterhalten. Zum anderen spielt aber auch die Übertragungsgeschwindigkeit eine Rolle: Allein die Tatsache, dass man innerhalb von Minuten reagieren bzw. eine Antwort erhalten kann, während Briefe erst am nächsten Tag ankommen, sorgt für eine höhere Austauschfrequenz und damit für mehr Aufwand pro Zeiteinheit. Diese Effekte summieren sich offenbar zu einem Rebound-Effekt von über 100\%. (Zum Rebound-Effekt siehe auch [Bin01], Kapitel 5 in [HBB03] sowie [HKS05].) 


\subsection{Langsamer arbeiten mit schnelleren PCs}

Die Effizienz der PC-Hardware als Rechenleistung pro Geld-, Volumen- oder Masseneinheit nimmt, wie oben erwähnt, laufend zu. Für die Benutzer/innen äußert sich dieser Fortschritt konkret darin, dass nach jedem Ersatz eines PCs nach durchschnittlich 2-3 Jahren ein wesentlich leistungsfähigeres Modell auf dem Schreibtisch steht, in der Regel mit einer neuen Version von Betriebssystem und Anwendungssoftware.

In einem gemeinsamen Projekt mit der Schwedischen Königlich-Technischen Hochschule (KTH) hat meine Forschungsgruppe die Frage untersucht, wie diese Entwicklung sich auf die Arbeiteffizienz der Benutzer/innen auswirkt [HKS05, Den05]. In einem Laborversuch wurde die Zeit gemessen, die 42 Versuchspersonen benötigten, vorgegebene Aufgaben zur Dateiverwaltung und Textverarbeitung mit unterschiedlich leistungsfähigen PCs zu erledigen.

Für das Experiment haben wir die im geschäftlichen Umfeld meistverbreiteten PC-Konfigurationen der Jahre 1997, 2000 und 2003 rekonstruiert (siehe Tabelle). Die technischen Daten zeigen den eindrucksvollen Fortschritt auf der Ebene der Hardware.

\begin{tabular}{|l|l|l|l|}
\hline \multicolumn{4}{|l|}{ Eigenschaften der verwendeten PC-Systeme (Quelle: Empa) } \\
\hline System & A & B & C \\
\hline Jahr & 1997 & 2000 & 2003 \\
\hline Taktfrequenz & $233 \mathrm{MHz}$ & $801 \mathrm{MHz}$ & $1,992 \mathrm{MHz}$ \\
\hline Arbeitsspeicher & $64 \mathrm{MB}$ & $128 \mathrm{MB}$ & $256 \mathrm{MB}$ \\
\hline Hintergrundspeicher & $2 \mathrm{~GB}$ & $20 \mathrm{~GB}$ & $56 \mathrm{~GB}$ \\
\hline Betriebssystem & Microsoft Windows & $\begin{array}{l}\text { Microsoft Windows } \\
2000\end{array}$ & $\begin{array}{l}\text { Microsoft Windows } \\
\text { XP }\end{array}$ \\
\hline Textverarbeitung & Microsoft Word 97 & $\begin{array}{l}\text { Microsoft Word } \\
2000\end{array}$ & $\begin{array}{l}\text { Microsoft Word } \\
2002\end{array}$ \\
\hline
\end{tabular}

\section{Methode}

Die Aufgaben bestanden in einfachen PC-basierten Aktivitäten, wie sie im Büroalltag häufig vorkommen (darunter das Kopieren, Verschieben, Auffinden und Umbenennen von Dateien, das Ersetzen und Formatieren von Text und das Positionieren von Abbildungen in einem Textdokument). Die Aufgaben waren so definiert, dass sie mit allen drei Systemen lösbar waren ${ }^{3}$ und außerdem die Hardware spürbar

3 Die Untersuchung sagt somit nichts darüber aus, wie die Verfügbarkeit neuer Funktionen, die auf den älteren Systemen noch nicht existierten, sich auf die Arbeitseffizienz auswirkt. Wir gehen jedoch davon aus, dass die gewählten Aufgaben Tätigkeiten entsprechen, die auch heute und in Zukunft einen erheblichen Teil der typischen PCbasierten Bürotätigkeit ausmachen. 
belasteten (eingebundene Fotografien, sehr lange Textfiles).

Den Versuchspersonen wurden zwei Aufgaben gestellt, im Folgenden ,file handling“ und „text editing“ genannt, die sie mehrfach und möglichst schnell an den drei Systemen auszuführen hatten. Die verschiedenen Ausprägungen einer Aufgabe waren strukturell gleich. Bezeichnungen, Seitenzahlen usw. waren jedoch verändert, um reine Erinnerungseffekte beim Wiederholen der Aufgaben auszuschließen. Die Zeit für das Lesen der Instruktion und für die jeweils erste Ausführung einer Aufgabe auf einem System wurden in der Auswertung nicht berücksichtigt. Die Reihenfolge der Systeme war zufällig gewählt und das Umschalten erfolgte mit einem Switchboard im Hintergrund, so dass kein Wechsel von Bildschirm, Tastatur oder Maus nötig war.

Um außerdem einen Effekt der Erwartungen der Versuchspersonen auf ihre Leistung auszuschließen, wurden zwei gleich große Gruppen gebildet. Die Mitglieder der einen Gruppe wurden beim Umschalten des Systems jeweils deutlich darüber aufgeklärt, dass es sich um einen „,sehr alten“, einen ,alten“ bzw. einen „neuen“ Computer handle, den sie sogleich benutzen würden. Die andere Gruppe wurde lediglich darüber informiert, dass es sich um drei unterschiedliche Systeme handle, die für den Versuch „modifiziert“ worden seien. Beim Umschalten wurde jeweils der Wechsel zu einem anderen Computer angekündigt.

\section{Resultate}

Abbildung 1 (c und d) zeigt die Ergebnisse des Experiments, ausgedrückt in erfolgreich gelösten Aufgaben pro Stunde. In Abbildung 1 oben (a und b) sind zum Vergleich die Anzahl der Komponenten der drei CPUs und die gemessenen Rechenleistungen der drei Systeme in MIPS angegeben.

Die ,file handling“-Aufgabe wurde auf dem 2000er-System etwas schneller gelöst (statistisch nicht signifikant) und auf dem 2003er-System wieder signifikant langsamer. Die ,text editing“-Aufgabe wurde ebenfalls mit dem zweitneuesten System am schnellsten gelöst. Statistisch signifikant ist hier nur die Verbesserung der Leistung beim Übergang 1997er- auf das 2000er-System. Zwischen den beiden Gruppen, deren Erwartungen im Versuch unterschiedlich geprägt worden waren, wurden keine signifikanten Unterschiede festgestellt.

Das Ergebnis zeigt insbesondere, dass der Übergang von Windows $2000 \mathrm{zu}$ Windows XP trotz einer ungefähr doppelten Rechenleistung nicht zu einer effizienteren Erledigung der Aufgaben führen muss, sondern sogar zu einem signifikanten Rückgang der durchschnittlichen Arbeitseffizienz führen kann. Dieses Ergebnis war besonders überraschend, weil die Versuchspersonen nach eigenen Angaben mit Windows 2000 mit Abstand die geringste Erfahrung hatten. Am Arbeitsplatz der Versuchspersonen war das System außerdem nie eingesetzt worden. Vielmehr war einige Monate vor dem Experiment ein Übergang von Windows NT zu Windows XP vollzogen worden. Das Ergebnis ist also nicht durch unterschiedliche Vertrautheit der Versuchspersonen mit den Systemen zu erklären. 


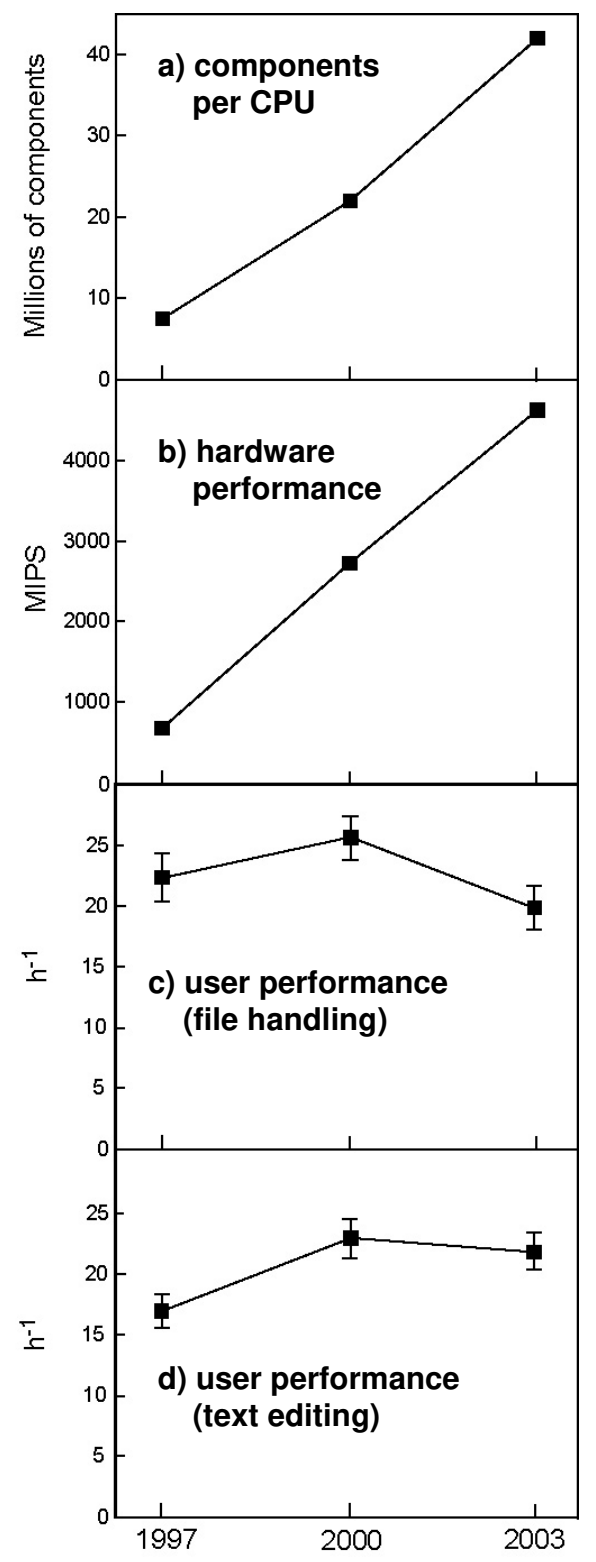

Abbildung 1. Hardware-Eigenschaften und Leistung der Versuchspersonen. a) Anzahl der Komponenten der CPU des jeweiligen PCs. b) Gemessene Leistung der CPU in Millionen Instruktionen pro Sekunde. c) Leistung der Versuchspersonen, ausgedrückt als Anzahl der erfüllten „file handling“-Aufgaben pro Stunde. d) Leistung der Versuchspersonen, ausgedrückt als Anzahl der erfüllten „,text editing“-Aufgaben pro Stunde. Die Fehlerbalken zeigen die Standardabweichung. (Quelle: Empa) 
Abbildung 2 zeigt eine nähere Analyse der Ergebnisse. Dabei wurde als Indikator für den Aufwand des PCs die Rechenzeit (Integral der Prozessorauslastung) und als Indikator für den Aufwand der Versuchsperson die Anzahl der Mauspositionierungen betrachtet. Im oberen Bild (Aufgabe „file handling“) ist zu erkennen, dass der Aufwand für Mensch und Maschine beim Übergang von 1997 zu 2000 abnimmt, aber - ebenfalls für Mensch und Maschine - beim Übergang zu 2003 wieder zunimmt. Auch im unteren Bild (Aufgabe „text editing“) nimmt die benötigte Rechenzeit in diesem Schritt zu, obwohl der Benutzer hier etwas weniger aktiv ist und also auch seltener Aktivitäten der Maschine auslöst. Die Zunahme der benötigten Rechenzeit ist hier besonders überraschend, da allein aufgrund der doppelten Prozessorleistung eigentlich eine Halbierung zu erwarten wäre. Auch wenn man annimmt, dass der höhere Rechenzeitbedarf zum Teil neuen Softwarearchitekturen geschuldet ist, die anderweitige (in unserem Experiment nicht zum Tragen kommende) Vorteile bieten, so ist es doch erstaunlich, dass dadurch der Effizienzfortschritt der Hardware offenbar nicht nur kompensiert, sondern sogar überkompensiert werden kann.

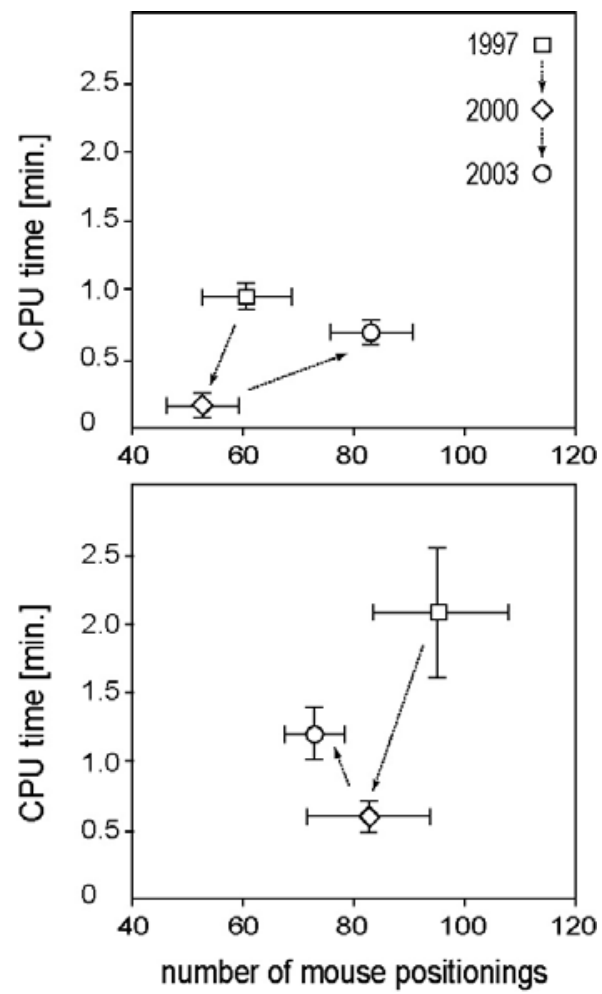

Abbildung 2. Aufwand der Versuchsperson, repräsentiert durch die Anzahl der vorgenommenen Mauspositionierungen, und der Aufwand der Computerhardware, ausgedrückt in Rechenzeit. a) Aufgabe „file handling“. b) Aufgabe „text editing“. Die Fehlerbalken zeigen die Standardabweichung. (Quelle: Empa) 


\section{Diskussion}

Welche Bedeutung dieses ernüchternde Ergebnis für die Praxis hat, ist unter anderem davon abhängig, wie weit die gestellten Aufgaben für den Büroalltag repräsentativ sind. Wir können nicht ausschließen, dass anderes Versuchsmaterial zu anderen Resultaten geführt hätte. Jedoch waren die Tätigkeiten der Versuchspersonen nicht ungewöhnlich und nutzten hauptsächlich grundlegende Funktionen von Betriebssystem und Textverarbeitungssoftware. Wir ziehen aus dem Experiment die folgenden Schlüsse:

- Der Wechsel zu einem leistungsfähigeren PC mit einer neuen Softwareversion führt nicht notwendigerweise zu einer höheren Arbeitseffizienz.

- Es gibt Fälle, in denen nach diesem Wechsel sowohl der Mensch als auch die Maschine mehr Zeit aufwenden müssen, um die gleichen Aufgabe zu lösen.

- Dies gilt unabhängig von Lerneffekten; die Lernphase beim Wechsel zu einem neuen System hat einen zusätzlichen Einfluss auf die Arbeitseffizienz, der hier nicht untersucht wurde.

Wir vermuten, dass das Ergebnis durch folgende Rebound-Effekte auf der Seite der Software-Entwickler zu erklären ist:

- „Featurism”: Höhere Hardware-Effizienz ermöglicht eine Ausweitung der Funktionalität von Softwareprodukten. Die Entwickler nutzen diesen Freiraum, um zusätzliche Funktionen einzuführen, ohne dabei auf die konzeptuelle Struktur des Systems hinsichtlich Einfachheit, Kompaktheit und Stabilität Rücksicht zu nehmen. Dadurch kann die durchschnittliche Zeit, die der Benutzer benötigt, um auf eine gegebene Funktion zuzugreifen, stark ansteigen. Die Auswertung der Videobänder aus dem Experiment stützt diese Erklärung: Die Versuchspersonen haben relativ viel Zeit damit verbracht, auf Funktionen zuzugreifen, die in einem älteren System direkter (d.h. mit weniger Mauspositionierungen) erreichbar waren.

- „Software Bloat”: Höhere Hardware-Effizienz verleitet Software-Entwickler zu ineffizienten Implementationen (in Bezug auf Rechenzeit oder Speicherplatz). Hierfür gibt es einige Evidenz in den Log-Files aus dem Experiment; es ist jedoch zu beachten, dass das Versuchsdesign nicht dafür ausgelegt war, diesen Aspekt im Detail zu untersuchen.

Mit Sicherheit beruhen viele Funktionserweiterungen und neue Softwarearchitekturen auf Anforderungen des Marktes, so dass nicht einfach eine Mentalität der ,grundlosen Verschwendung“ auf Seite der Entwickler unterstellt werden kann. Dennoch sollte man erwarten, dass dies für die Benutzer/innen, die mit einem Softwareprodukt primär die gleichen alltäglichen Handlungen ausführen wollen wie mit der Vorgängerversion, wenigstens keine gravierenden Nachteile mit sich bringt.

Die Ergebnisse unserer Untersuchung lassen nicht den Schluss zu, dass es keinen Effizienzfortschritt durch die Erneuerung von Hardware und Software im Büroalltag gäbe. Das Laborexperiment hat eine künstliche Situation geschaffen, in der die Versuchspersonen ein exakt vorgegebenes Ergebnis zu reproduzieren hatten. Der Output war also fixiert, und nur der Input (in Form von Zeitaufwand) konnte 
variieren. In einer realen Situation haben Benutzer/innen dagegen mehr Gestaltungsfreiheit und können neue technische Möglichkeiten dazu nutzen, qualitativ andere Outputs zu erzeugen, z.B. Dokumente mit einem ausgefeilteren Layout. Abhängig davon, wie man diese qualitative Veränderung im Output bewertet, wird sich auch die Effizienz anders darstellen. Auch ist zu berücksichtigen, dass durch Fortschritte in der Hardwareeffizienz - soweit sie nicht durch ineffiziente Implementationen kompensiert wurden - neue Arbeitsstile möglich geworden sind (etwa paralleles Bearbeiten vieler gleichzeitig geöffneter Dokumente), die früher an technische Grenzen gestoßen wären. Die Möglichkeit zur individuellen Gestaltung der Arbeitsabläufe schafft grundsätzlich Freiräume für Effizienzverbesserungen.

Insgesamt ist deshalb zu erwarten, dass die individuelle Arbeitseffizienz bei PCbasierten Tätigkeiten mit dem technischen Fortschritt zunimmt, wenngleich dieser Fortschritt aufgrund der beschriebenen Rebound-Effekte im Software-Design offenbar geringer ausfällt als prinzipiell möglich.

Ein häufig vorgebrachter Einwand gegen unsere Kritik am Software-Design lautet, dass der Markt ein solches Problem beheben müsste, wenn die Kunden wirklich unzufrieden sind. Dagegen ist einzuwenden, dass der Markt im Bereich der IKT weitgehend versagt, weil die notwendige Kompatibilität bzw. Interoperabilität fast zwangsläufig zur Marktdominanz einzelner Hersteller führt. Wir haben in unserem Experiment nicht nur Unzufriedenheit beobachtet, sondern auch Stress und - in Einzelfällen - ernsthafte Verzweiflung. Wir gehen davon aus, dass die jeweiligen Benutzer/innen ihre Unzufriedenheit im Alltag verbergen in der Annahme, alle anderen seien in der Lage, effizienter mit der gleichen Technologie umzugehen. Außerdem haben die meisten Benutzer/innen kaum Vergleichsmöglichkeiten und keine freie Wahl, da der Arbeitgeber über die technische Infrastruktur entscheidet.

Was kann man daraus für eine zukünftige „Informatisierung des Alltags“ lernen? Offenbar ist es nicht selbstverständlich, dass die durch den IKT-Fortschritt geschaffenen Freiräume von den Entwicklern so genutzt werden, dass die Benutzer/innen ihre Ziele damit besser erreichen können. Nicht auszudenken, dass die gleiche Design-Mentalität, die die heutige PC-Software prägt, im Zeitalter der „smart objects" unser gesamtes Alltagsleben durchdringen könnte!

Ein fairer Wettbewerb könnte die Entwicklung grundsätzlich in eine wünschbare Richtung lenken. Die „Informatisierung des Alltags“ wird sich aber nicht in einem idealen Markt vollziehen, sondern in realen ökonomischen Strukturen mit wenigen dominanten Herstellern. 


\subsection{Rebound-Effekte in Organisationen}

Wir haben bisher die Interaktion zwischen Mensch und Computer betrachtet und das organisatorische Umfeld ausgeblendet. Auf der Ebene der Organisation aber sind vielleicht die wichtigsten Rebound-Effekte zu beobachten.

\section{Vorbemerkungen zu Effizienz und Produktivität}

Betrachtet man einen beliebigen Transformationsvorgang als Produktion im ökonomischen Sinne (was prinzipiell immer möglich ist), so fallen Effizienz (wie oben in 2.1 definiert) und Produktivität zusammen. Produktivität ist definiert als Output pro Einsatz von Produktionsfaktoren. Begrenzt man die Betrachtung auf einen einzelnen Produktionsfaktor, z. B. Arbeit, so spricht man von Faktorproduktivität, im diesem Fall von Arbeitsproduktivität. Input und Output werden in der Regel nicht in physikalischen Einheiten, sondern durch ihren ökonomischen Wert repräsentiert.

\section{Das IT-Produktivitätsparadox}

Seit den späten 1980er-Jahren wird vor allem in der ökonomischen Literatur die Beobachtung diskutiert, dass die mit dem Einsatz von Informationstechnologien verfolgten Produktivitätsziele nicht erreicht werden. Dieses „IT-Produktivitätsparadox" "4 wurde 1987 von Robert Solow erstmals thematisiert. Der Nobelpreisträger für Ökonomie initiierte die Debatte mit seinem viel zitierten Satz: „We see the computer age everywhere except in the productivity statistics" [Sol87]. Die darauf folgende Kontroverse motivierte eine Reihe interessanter Forschungsarbeiten über die Frage, wie IT die Produktivität von Organisationen beeinflusst.

Das IT-Produktivitätsparadox wird vor allem im Kontext des Dienstleistungssektors diskutiert. ${ }^{5}$ In modernen Dienstleistungsunternehmen wird die Wertschöpfung typischerweise durch Personen erbracht, deren Arbeit von einem Computer unterstützt wird. Angestellte von Banken und Versicherungen, Reisebüros, Rechtsanwaltskanzleien, Verlagen, Forschungsinstituten - um nur einige zu nennen verbringen einen großen Teil ihrer Arbeitszeit vor einem PC. Das ist immer häufiger auch während Sitzungen oder in Kundengesprächen der Fall.

Die Produktivität einer typischen Dienstleistungsorganisation ist deshalb - vereinfacht ausgedrückt - das Aggregat der Produktivität von Menschen, die einen PC benutzen, also ein aggregiertes Ergebnis jener Art von Arbeitseffizienz, die wir oben diskutiert haben. Die empirisch belegte Tatsache, dass technische Effizienz nicht zwingend die individuelle Arbeitseffizienz fördert, ist folglich eine mögliche Erklärung für das Produktivitätsparadox. Diese Erklärung ist jedoch nicht aus-

4 Engl. „IT productivity paradox”. Der Beginn der Debatte fällt in eine Zeit, in der Informationstechnologie und Kommunikationstechnologie noch getrennte Bereiche waren, deshalb verwendet man in diesem Kontext noch die Bezeichnung „IT” (statt „IKT” bzw. „ICT”).

5 Dass die (weitgehend IT-basierte) Automatisierung der industriellen Produktion zu Produktivitätsfortschritten geführt hat, steht nicht zur Diskussion. 
reichend. Zwei weitere - vielleicht die entscheidenden - Rebound-Effekte sind auf der organisatorischen Ebene zu finden.

\section{Zielverschiebung}

Die Tatsache, dass sich durch den informationstechnischen Fortschritt gewisse Ziele der täglichen Arbeit leichter und billiger erreichen lassen als früher (beispielsweise ein fehlerfreies Dokument), führt zu einer Verschiebung der Motivation in Richtung solcher Ziele. Der Soziologe Paul Attewell beschreibt diesen als Zielverschiebung (goal displacement) bezeichneten Effekt am Beispiel der Textverarbeitung:

„Studies of individuals using word processors have noted that instead of using the technology to produce more documents in a given length of time, employees make five times as many corrections as previously. They also pay more attention to fonts, graphics and so on. In other words, at this individual level, there is a displacement from the goal of increasing throughput productivity to the goal of enhancement of quality and appearance“" [Att93, p.4]

Dieser Effekt ist aber nicht allein auf der individuellen Ebene zu erklären. Vielmehr ist es erst die Konkurrenz innerhalb der Organisation, die der kollektiven Zielverschiebung zum Durchbruch verhilft. Die technischen Möglichkeiten setzen faktische Qualitätsstandards, die möglicherweise nicht im Interesse einer produktiven Organisation liegen. Die Führung der Organisation könnte dies nur durchbrechen, indem sie die Standards nach unten korrigiert. Um im Beispiel zu bleiben: Die Unternehmensleitung könnte es verbieten, Manuskripte in einem anderen Format als ASCII zu verfassen, E-Mails im html-Format zu verschicken, animierte Präsentationen zu erstellen usw. - die Möglichkeiten für solche ,produktiven Beschränkungen" sind nahezu unbegrenzt.

Mit etwas Phantasie lässt sich leicht ausmalen, welche Möglichkeiten zu Zielverschiebungen eine von ,smart objects“ durchdrungene Welt eröffnen wird.

\section{Der Bürokratie-Rebound-Effekt}

Die Kosten für das Dokumentieren von Tätigkeiten nehmen durch den IT-Fortschritt laufend ab. Man sollte also erwarten, große Organisationen würden sich dank IT von ihrer sprichwörtlichen Bürokratie befreien und ihrer Belegschaft würde dann mehr Zeit für produktive Tätigkeiten bleiben. Leider ist meist das Gegenteil der Fall: Weil die interne Nachfrage nach dokumentierender Information potenziell unbegrenzt ist, führt jede Kostensenkung zu einem unvermeidlichen Rebound-Effekt: Je effizienter dokumentiert werden kann, desto mehr Dokumentationspflichten werden eingeführt. Dies führt zu einem Wachstum unproduktiver, größtenteils legitimativer Tätigkeiten, das mit älterer Technik undenkbar gewesen wäre. Gemäß Attewell [Att93] kann dieser Rebound-Effekt $100 \%$ übersteigen, d.h. die Gesamtkosten für die Verarbeitung interner Information in Organisationen können mit IT höher sein als ohne IT.

Wenn dies bereits mit herkömmlicher IT geschieht - was ist erst zu erwarten, wenn dank schlauer Alltagsgegenstände alles messbar und dokumentierbar zu werden scheint? 


\subsection{Schlussfolgerungen}

Wie der Rückblick in die jüngere Geschichte der IKT zeigt, ist es keineswegs selbstverständlich, dass durch Fortschritte auf der technischen Ebene auch die mit dem IKT-Einsatz intendierten Ziele besser erreicht werden. Selbst relativ klar definierte Ziele wie Effizienz und Produktivität konnten - trotz einer technischen Effizienzverbesserung von ungefähr einem Faktor 1000 in zwei Jahrzehnten - nicht im erwarteten Ausmaß erreicht werden.

Der Grund hierfür ist die verengte Perspektive technikzentrierter Visionen, die das Umfeld und damit entscheidende Nebenwirkungen ausblendet: ReboundEffekte im Software-Design, bei der individuellen Anwendung und in der Organisation.

Solange die Auswirkungen des IKT-Einsatzes auf Mensch und Organisation nicht besser verstanden sind (und hierfür gibt es brauchbare Ansätze), sind Visionen einer „Informatisierung des Alltags“ unvollständig und geradezu irreführend. Die Interaktion von Mensch, Technik und Organisation muss in ihrer Dynamik beachtet werden, wenn die Entwicklung gestaltbar sein soll.

\section{Risiken der „Informatisierung des Alltags“}

Eine technikzentrierte Vision der „Informatisierung des Alltags“ ist „Pervasive Computing“, kurz „PvC“. IKT-Komponenten werden nach dieser Vision ihre Dienste an jedem Ort und zu jeder Zeit zur Verfügung stellen. Gebrauchsgegenstände, Fahrzeuge, Gebäude, Kleidung und teilweise auch der menschliche Körper werden mit Komponenten ausgestattet, die in der Lage sind, Daten zu speichern, zu verarbeiten und zu übertragen.

Im Jahr 2002 erhielt meine Forschungsgruppe an der Empa zusammen mit dem Institut für Zukunftsstudien und Technologiebewertung (IZT) in Berlin vom Schweizerischen Zentrum für Technologiefolgen-Abschätzung (TA-SWISS) den Auftrag, die Chancen und Risiken des PvC für Gesundheit und Umwelt abzuschätzen. Die 2003 veröffentlichte Studie [HBB03] wurde im Auftrag des Europäischen Parlaments überarbeitet und ins Englische übersetzt [HBB05], eine Kurzfassung außerdem in alle Amtssprachen der Europäischen Union. Die Zeitschrift „Human and Ecological Risk Assessment" widmete dem Thema eine Special Section [SHR04], das „IEEE Technology and Society Magazine“ ein Special Issue [BCH05].

Das große Interesse an Technologiefolgen-Abschätzung für $\mathrm{PvC}$ zeigt unseres Erachtens ein verbreitetes Unbehagen gegenüber einer technisch-ökonomisch determinierten Entwicklung im IKT-Markt, die keinen Entscheidungsspielraum, keinen Gestaltungsspielraum mehr offen zu lassen scheint, obwohl sie doch im Zeichen unbegrenzter technischer Möglichkeiten propagiert wird.

Die TA-SWISS-Studie war ein erster Ansatz, zukünftige Entwicklungspfade für $\mathrm{PvC}$ und insbesondere die damit verbundenen Risiken aufzuzeigen. Der Fokus lag auf gesundheitlichen, ökologischen und sozialen Risiken. 


\subsection{Pervasive Computing als gesellschaftlicher „Selbstversuch“}

Die Technologiefolgen-Abschätzung ist im Normalfall weit davon entfernt, zukünftige Risiken quantifizieren zu können. Es ist zwar möglich, Anwendungsszenarien für vorhersehbare Technologien im Sinne möglicher Entwicklungspfade zu konstruieren - welches Szenario tatsächlich eintreten wird, ist aber nicht prognostizierbar. Gerade weil die Technologien (oder zumindest die Art ihrer Anwendung) neu sind, ist es nicht möglich, aus der Vergangenheit in die Zukunft zu extrapolieren. Bestenfalls - und das haben wir oben in Abschnitt 2 versucht - kann man aus der Geschichte lernen, was bisher schief gelaufen ist und worauf man deshalb in Zukunft besonders achten sollte.

Um die medizinische Analogie wieder aufzugreifen: Die Gesellschaft ist in der gleichen Lage wie ein Patient, der ein Medikament einnehmen soll, mit dem noch keine Erfahrungen vorliegen. Der breite Einsatz von Technologien, die zuvor nur in einem eng beschränkten Rahmen getestet werden konnten, ist in diesem Sinne ein „Selbstversuch” der Gesellschaft. Die damit verbundenen Risiken gehen wir der eine mehr, der andere weniger freiwillig - ein, um Chancen wahrzunehmen. Im Falle der Mobiltelefonie läuft dieser Versuch schon einige Jahre, und bisher sind keine gravierenden Probleme aufgetreten.

Pervasive Computing ist wesentlich komplexer als Mobiltelefonie. Der vielleicht entscheidende Unterschied ist die Auflösung der Grenzen zwischen IKT und Nicht-IKT. Während wir heute noch deutlich einen PC, ein Handy oder einen PDA als „IKT“ erkennen und ein Buch, einen Tisch, eine Brille als „Nicht-IKT“, so wird dieser Unterschied in einer PvC-Welt faktisch verschwinden. Folglich wird auch das Erzeugen von Daten (die gespeichert und übertragen werden können) immer häufiger als beiläufige, nicht bewusst vorgenommene Handlung auftreten. Tastatur und Display werden nicht mehr als identifizierende Merkmale für IKT taugen, weil eingebettete Mikrochips auf anderen Kanälen mit ihrer Umgebung kommunizieren werden. Die normale Benutzung von Alltagsgegenständen wird (über Sensoren) die Eingabedaten für eingebettete Prozessoren generieren, und ebenso wird die Ausgabe über Aktoren in diesen Objekten erfolgen. Der größte Teil des Datenverkehrs wird durch Maschine-Maschine-Kommunikation verursacht werden, d.h. nicht mehr direkt von Menschen initiiert sein, wie es heute z.B. beim Versenden von E-Mails der Fall ist.

\subsection{Das Vorsorgeprinzip in der Informationsgesellschaft}

Entscheidungen, die auf nicht quantifizierbare Risiken Bezug nehmen, lassen sich nicht vollständig objektivieren. Weil politische Entscheidungen aber auch gefällt werden müssen in Bereichen, in denen der Stand des Wissens nicht ausreicht, um quantitativ exakte Prognosen zu stellen, sind Handlungsmaximen notwendig, um mit solchen Situationen umzugehen. Eine solche Maxime ist das Vorsorgeprinzip (engl. precautionary principle), das ursprünglich aus dem Umweltbereich stammt.

Das Vorsorgeprinzip dient dem Umgang mit Risiken in Situationen, in denen keine akute Gefährdung gegeben ist. Es hat den Zweck, auch solche Risiken zu 
minimieren, die sich möglicherweise erst langfristig manifestieren, und Freiräume für zukünftige Entwicklungen zu erhalten.

Vorsorge (engl. precaution) unterscheidet sich damit von der Gefahrenabwehr (engl. prevention), die im Falle eines Risikos mit akutem Gefährdungspotenzial zur Anwendung kommt. Ein Risiko muss nachgewiesen und ausreichend hoch sein, um als Gefahr im juristischen Sinne zu gelten.

Warum sollte es geboten sein, Einwirkungen auch dann zu vermeiden, wenn von ihnen keine akute Gefährdung ausgeht? In der juristischen Literatur werden zwei Theorien des Vorsorgeprinzips (VSP) diskutiert [Köc89, Bey92]:

- Die Ignoranz-Theorie rechtfertigt eine vorsorgliche Belastungsminimierung durch die Begrenztheit des Wissens (Ungewissheit) über zukünftige Wirkungen. Das VSP soll den vernünftigen Umgang mit ungeklärten Risiken unterstützen, während die Gefahrenabwehr den Umgang mit sicheren, d.h. ausreichend nachgewiesenen Risiken regelt.

- Die Freiraum-Theorie besagt, dass mit dem VSP Freiräume für die Aktivitäten zukünftiger Generationen erhalten werden sollen.

Nach Van den Daele [Dae01] wird das VSP in Anspruch genommen, um die Technikentwicklung stärker in den Einflussbereich von Politik und Öffentlichkeit zu bringen. Dies erscheint notwendig, weil der technische Fortschritt nicht nur Naturgefahren in den Verfügungshorizont menschlicher Entscheidungen holt, sondern gleichzeitig auch neue Risiken schafft. Neue Technologien können inakzeptable Nebenwirkungen für die Gesellschaft haben. Das VSP soll gewährleisten, dass sich die Gesellschaft sich bewusst für oder gegen das Eingehen solcher Risiken entscheiden kann, und zwar auch dann, wenn über die Existenz und das Ausmaß eines Risikos Ungewissheit besteht.

Ungeklärte Risiken können nicht quantitativ bewertet werden. Auch wenn sich in einigen Fällen das Schadensausmaß grob abschätzen lässt, ist die Eintrittswahrscheinlichkeit nicht bestimmbar. In der Risikoforschung werden deshalb eine Reihe von Kriterien zur qualitativen Charakterisierung von Risiken diskutiert.

Um das Vorsorgeprinzip für Fragestellungen der Informationsgesellschaft zu präzisieren, haben wir in der TA-SWISS-Studie die folgenden Kriterien verwendet:

- Sozioökonomische Irreversibilität: Ist die Wiederherstellung des Ausgangszustandes aus wirtschaftlichen oder rechtlichen Gründen praktisch unmöglich?

- Verzögerungswirkung: Ist die Verzögerung zwischen dem Eintreten der Ursache und dem Auftreten des Schadens groß? ${ }^{6}$

- Konfliktpotenzial mit den beiden Unterkriterien

○ Freiwilligkeit: Wird das Risiko freiwillig eingegangen?

○ Fairness: Sind Nutzen und möglicher Schaden gleich verteilt? ${ }^{7}$

6 Eine Verzögerung - im Verhältnis zur Ausbreitungsgeschwindigkeit der Ursache - ist ungünstig, weil dann Gegenmaßnahmen im Rahmen des Schutzprinzips (Gefahrenabwehr) erst spät einsetzen. Verzögerungswirkung ist also ein zentrales Kriterium unter dem Aspekt des Vorsorgeprinzips.

7 Menschen gehen Risiken ein, um Chancen wahrzunehmen. Wenn jedoch die Chance (der potenzielle Nutzen) von anderen Akteuren wahrgenommen wird als von jenen, die das Risiko (den potenziellen Schaden) tragen, ist das Konfliktpotenzial hoch. 
- Belastung für die Nachwelt: Verschlechtert der eingetretene Zustand die Möglichkeiten zukünftiger Generationen, ihre Bedürfnisse zu befriedigen?

Zur näheren Erläuterung und Begründung dieser Kriterien sowie zur Methodik ihrer Anwendung verweisen wir auf die Originalstudie [HBB03] und darauf aufbauende Veröffentlichungen [HSK04, SHR04].

\subsection{Ergebnisse der TA-SWISS-Studie}

Die Studie hat auf der Basis von Literaturrecherchen, Szenarien und Expertenworkshops 31 gesundheitliche, ökologische und soziale Auswirkungen von PvC identifiziert, die entweder als Chance, als Risiko oder als ambivalent (Nutzen oder Schaden sind von weiteren Faktoren abhängig) klassifiziert wurden. Durch Anwendung der oben genannten qualitativen Kriterien wurden 11 Risiken höherer Priorität herausgefiltert, die schließlich zu Problemfeldern (Risiko-Clustern) verdichtet wurden. Ohne auf die Zwischenschritte im Detail eingehen zu können, fassen wir hier die wichtigsten Problemfelder zusammen (die Reihenfolge bedeutet keine Priorisierung): ${ }^{8}$

- Stress: PvC kann aus mehreren Gründen Stress auslösen, darunter schlechte Benutzbarkeit der Systeme, Störung und Ablenkung der Aufmerksamkeit, das Gefühl des Überwachtwerdens (Privatsphäre/Datenschutz), möglicher krimineller Missbrauch sowie steigende Anforderungen an die Produktivität des Einzelnen. Stress ist ein wichtiger Einflussfaktor für die Gesundheit.

- Unfreiwilligkeit: Ein Teil der Konsumenten könnten durch die Entwicklung in Richtung PvC in eine Lage gebracht werden, in der sie diese Technologie unfreiwillig anwenden müssen (z.B. weil Alternativen nicht mehr angeboten werden) oder unfreiwillig mitfinanzieren (z.B. über steigende Krankenkassenbeiträge).

- Verursacherprinzip: Die Ursachen von Schäden, die durch das Zusammenwirken mehrerer Komponenten aus Computerhardware, Programmen und Daten in Netzwerken entstehen, sind in der Regel nicht aufzuklären, weil die Komplexität dieser verteilten Systeme weder mathematisch noch juristisch beherrschbar ist. Da mit PvC die Abhängigkeit von solchen Systemen zunehmen wird, ist insgesamt ein Anstieg des durch unbeherrschte technische Komplexität entstehenden Schadens zu erwarten. Die Folge ist, dass ein wachsender Teil des Alltagslebens sich faktisch dem Verursacherprinzip entzieht.

- Ökologische Nachhaltigkeit: Der Verbrauch seltener Rohstoffe durch die Produktion von Elektronik und der Stromverbrauch durch die stationäre Infrastruktur könnten stark zunehmen. Wenn die Entsorgung von Millionen winziger Komponenten als Elektronikabfall nicht adäquat geregelt werden kann, gehen wertvolle Rohstoffe verloren und gelangen Schadstoffe in die Umwelt (speziell zum letzten Punkt siehe auch [KWE05, WEH05, WOS05]).

8 Wir gehen hier nicht auf den Themenkomplex ,nicht-ionisierende Strahlung“ ein, der in der Studie ebenfalls ausführlich behandelt wurde. 
Im Sinne des Vorsorgeprinzips formuliert die Studie Maßnahmen an die Adresse von Politik, Bildung und Wirtschaft, die geeignet sind, diese Risiken frühzeitig - also bevor sie zu einer Gefahr werden - zu minimieren. Die Aussagen zu den Problemfeldern sind deshalb nicht als Prognose zu verstehen, sondern als Hinweise auf mögliche Fehlentwicklungen, die noch korrigiert werden können.

Als übergeordnete Maßnahme wird die Koordination der beiden Politikfelder Informationsgesellschaft und nachhaltige Entwicklung vorgeschlagen. Eine geeignete Ausgestaltung der Informationsgesellschaft ist eine notwendige Voraussetzung für die Möglichkeit einer nachhaltigen Entwicklung (siehe weiterführend auch [HAE06]).

\section{Kritische Fragen zur Informatisierung des Alltags}

Zusammenfassend möchte ich zur Vision einer „Informatisierung des Alltags“ sechs kritische Fragen formulieren, die in einem möglichst offenen Diskurs geklärt werden sollten:

- Wie können die durch den IKT-Fortschritt geschaffenen Freiräume im Interesse der Benutzer/innen ausgeschöpft werden, obwohl wegen des latenten Zielkonflikts zwischen Wettbewerb (der Vielfalt bedingt) und Standardisierung (die Einheitlichkeit bedingt) der IKT-Markt nur eingeschränkt funktioniert?

- Genügt unser Verständnis der Wechselwirkungen zwischen Mensch, Technik und Organisation (d.h. von sozio-technischen Systemen), um heutige und zukünftige IKT besser für eine erfolgreiche Entwicklung von Organisationen einzusetzen als bisher?

- Ist unser Entwicklungsstand in den Bereichen Mensch-Maschine-Schnittstellen, Datenschutz und Datensicherheit ausreichend und wird er ausreichend beachtet, damit die Informatisierung des Alltags keinen zusätzlichen psychischen Stress durch Ablenkung, Überwachung und drohenden Missbrauch mit sich bringen wird?

- Kann die Gesellschaft mit den Konflikten umgehen, die zwischen den freiwilligen Benutzer/innen und den nur teilweise freiwillig oder unfreiwillig Betroffenen einer ubiquitären Technologie entstehen könnten?

- Wird eine informationstechnisch vernetzte Alltagswelt, die eher als emergentes Phänomen denn als konstruiertes und beherrschbares System zu verstehen ist, in Schadensfällen noch die Ermittlung eines Verursachers zulassen? Ist unser Rechtssystem auf dieses Problem vorbereitet?

- Setzen die geltenden Rahmenbedingungen die richtigen Anreize, die „Informatisierung des Alltags“ für das Ziel einer nachhaltigen Entwicklung zu nutzen? Oder verleiten sie dazu, eine Infrastruktur aufzubauen, die neue Abhängigkeiten und Verletzlichkeiten schafft und dauerhaft mehr materielle und energetische Ressourcen beansprucht, als sie einspart? 


\section{Literatur}

[Att93] Attewell P (1993) Why productivity remains a key measure of IT impact. Proc. Productivity impacts of information technology investments conference, Charleston SC

[BCH05] Bütschi D, Courant M, Hilty LM (2005) Towards Sustainable Pervasive Computing. Guest Editorial. IEEE Technology and Society Magazine, 24, 2005 (1): 7-8.

[Bey92] Beyer H-M (1992) Das Vorsorgeprinzip in der Umweltpolitik. Schriftenreihe Wirtschafts- und Sozialwissenschaften, Bd. 10; Verlag Wissenschaft \& Praxis

[Bin01] Binswanger M (2001) Technological Progress and Sustainable Development: What About the Rebound Effect? Ecological Economics 36: 119-132.

[Dae01] Van den Daele W (2001) Zur Reichweite des Vorsorgeprinzips - rechtliche und politische Perspektiven. In: Lege, J. (Hrsg.): Gentechnik im nicht-menschlichen Bereich - was kann und was sollte das Recht regeln? Verlag Arno Spitz, Berlin: 101-125

[Den05] Denzler L (2005) Zwei Schritte vor, ein Schritt zurück: Software-Update-Zyklus lähmt Arbeitsproduktivität. Neue Zürcher Zeitung. 02.11.2005.

[HAE06] Hilty LM, Arnfalk P, Erdmann L, Goodman J, Lehmann M, Wäger P (2006) The Relevance of Information and Communication Technologies for Environmental Sustainability - a Prospective Simulation Study. Environmental Modelling \& Software, in press

[HBB03] Hilty LM, Behrendt S, Binswanger M, Bruinink A, Erdmann L, Fröhlich J, Köhler A, Kuster N, Som C, Würtenberger F (2003) Das Vorsorgeprinzip in der Informationsgesellschaft - Auswirkungen des Pervasive Computing auf Gesundheit und Umwelt. Herausgegeben vom Zentrum für Technologiefolgen-Abschätzung (TA-SWISS), Bern (TA 46/2003)

[HBB05] Hilty LM, Behrendt S, Binswanger M, Bruinink A, Erdmann L, Fröhlich J, Köhler A, Kuster N, Som C, Würtenberger F (2005b) The precautionary principle in the information society - effects of pervasive computing on health and environment. Second Revised Edition. Edited by the Swiss Center for Technology Assessment (TA-SWISS), Bern, Switzerland (TA46e/2005) and the Scientific Technology Options Assessment at the European Parliament (STOA 125 EN)

[HKS05] Hilty LM, Köhler A, Schéele F, Zah R, Ruddy T (2005) Rebound effects of progress in information technology. Poiesis \& Praxis: International Journal of Technology Assessment and Ethics of Science (11) : 1-20, DOI 10.1007/s10202-005-0011-2

[HSK04] Hilty LM, Som C, Köhler A (2004) Assessing the human, social and environmental risks of pervasive computing. Human and Ecological Risk Assessment. 102004 (5): $853-874$

[Köc89] Köchlin D (1989) Das Vorsorgeprinzip im Umweltschutzgesetz, unter besonderer Berücksichtigung der Emissions- und Immissionsgrenzwerte. Neue Literatur zum Recht, Hebling \& Lichtenhahn, Basel und Frankfurt am Main

[KWE05] Kräuchi P, Wäger PA, Eugster M, Grossmann G, Hilty LM (2005) End-of-Life Impacts of Pervasive Computing. IEEE Technology and Society Magazine 24 (1): 45-53

[Mat03] Mattern F (2003) Vom Verschwinden des Computers - Die Vision des Ubiquitous Computing. In: Mattern, F (Hrsg.): Total Vernetzt. Springer-Verlag: 1-41

[SHR04] Som C, Hilty LM, Ruddy T (2004) The Precautionary Principle in the Information Society. Human and Ecological Risk Assessment 10 (5): 787-799

[Sol87] Solow R (1987) We'd better watch out. Book Review 36, New York Times (July 12) 
[WEH05] Wäger, P, Eugster, M, Hilty, L M, Som, C (2005) Smart labels in municipal solid waste - a case for the precautionary principle? Environmental Impact Assessment Review. 25 (5): 567-586

[WOS05] Widmer R, Oswald-Krapf H, Sinha-Khetriwal D, Schnellmann M, Böni H (2005) Global perspectives on e-waste. Environmental Impact Assessment Review 25 (5): 436458 